EDITORIAL

For reprint orders, please contact: reprints@futuremedicine.com

\section{Could the detection of visual disturbances associated with Parkinson's disease genes in flies lead to new treatments for the disease?}
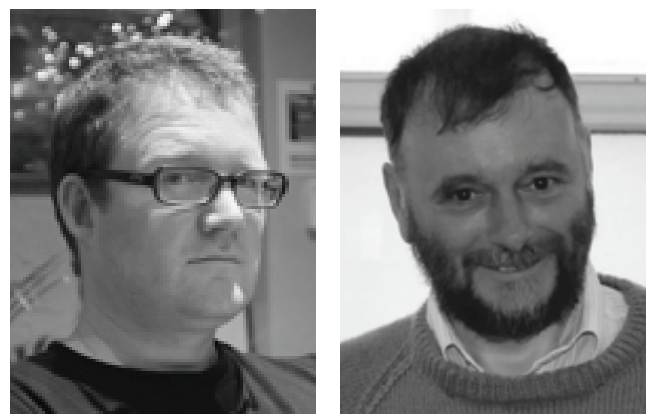

\author{
"Could studies of fly vision \\ really expedite the search for \\ new Parkinson's disease \\ therapies? Yes, here's how..."
}

Neurodegenerative Disease Management

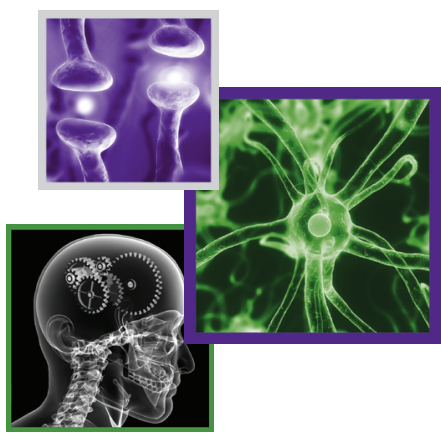

Alex R Wade1 \& Christopher JH Elliott ${ }^{*, 2}$

Could studies of fly vision really expedite the search for new Parkinson's disease therapies? Yes, here's how:

\section{What's the problem?}

Parkinson's disease (PD) is the secondmost common neurodegenerative disorder. Patients typically manifest with a serious reduction in movement - bradykinesia and/or hypokinesia. The current treatment focus is on L-DOPA (or another dopaminergic drug) which can relieve the symptoms [1]. However, this approach has two disadvantages: it does not slow the progression of the disease, and it induces (after 5 years of treatment) dyskinesia, characterized by involuntary jerky movements. At present there is no cure and the need for novel, disease-modifying therapies is urgent.

How can we find a model of PD to test drugs?

The discovery of inherited forms of PD caused by single gene mutations about 15 years ago, provided a major new experimental impetus (for review, see [2]). Once identified, the genes could be manipulated to generate cellular and animal models, in which causes and the effects of drugs could be analyzed experimentally. The most common PD-related mutation is in the gene for LRRK2, where a single amino-acid change G2019S (glycine $\rightarrow$ serine at position 2019) causes up to 5\% of all instances of PD. In the test tube, this change increases the kinase activity of the protein [3] but its normal cellular target(s) are still uncertain. To find these requires the development of effective animal models, and disappointingly, mouse models have failed to recapitulate many of the PD phenotypes [4]. We therefore chose to work with the fruit fly, Drosophila, because it has the most tractable genetics, high fecundity, and easy experimentation without requiring ethical permission [5]. Most importantly, Drosophila can be made to express both the normal wild-type human hLRRK2 gene (which has little effect), or the form associated with $\mathrm{PD}, L R R K 2$ G2019S (which causes clear physiological deficits).

$\mathrm{PD}$ is a disease of the dopaminergic system. Since flies and humans both have dopaminergic neurons in their visual systems, we asked if we could detect the effects 


\section{"Although flies are not 'little humans', they share many genes..."}

of the G2019S mutation through its effects on visual responses. To do this, we adapted a sensitive test of visual function that has been used for many years to study visual dysfunction in humans: the steady-state visually evoked potential (SSVEP).

When we used this type of recording technique in normal flies, we found that their responses resembled those seen in humans [6]. This is a key result, showing that the function of the visual system in flies and humans is extremely similar. This parallels the anatomical similarities first shown by the Nobel prize-winning anatomist Ramon y Cajal in 1913 [7].

\section{What happens to the visual system of flies} with PD mutations?

After discovering that the visual nervous system of the fly responded in the same way as the human, we then compared control flies (expressing the normal, wild-type gene, $h L R R K 2$, or non-transgenic flies) with those expressing LRRK2-G2019S in their dopaminergic neurons ('PD-mimic'). Old PD-mimic flies had almost no visual response, extensive degeneration of the retina and mitochondrial damage [8]. This was associated with two forms of cell death (apoptosis and autophagy). Degeneration was highly specific for the combination of dopaminergic neurons and the G2019S transgene. No other LRRK2 mutation had a similar effect. A significant breakthrough was the observation that neurodegeneration was accelerated by keeping the flies in a 'disco chamber', in which the lights were turned on or off repeatedly [8]. This provided the idea that the demand for energy in the PD-mimic flies might be at the root of the toxic cascade. This led us to hypothesize that the very young PD-mimic flies might have an overactive visual system, as a result of the increased LRRK2 kinase activity. We tested this in two ways: (i) by increasing neural activity genetically and finding faster degeneration $[8]$, and (ii) by showing directly a failure of neural gain control in young PD-mimic flies [6]. Because of the specificity of the SSVEP technique, we could characterize abnormalities in the first order photoreceptors, the second order lamina neurons and in the third/fourth order neurons in the medulla.

Do drugs affect the degeneration in this PD-mimic?

Having shown a clear PD-mimic phenotype, of initial hyperactivity, we fed two inhibitors, targeted at the kinase activity of LRRK2, to flies to see if we could rescue this $\mathrm{PD}$-mimic phenotype. One of these compounds, LRRK2-IN-1, provided an effective rescue, but we used the power of fly genetics to show this was accompanied by off-target side effects. A second compound, BMPPB-32, also reverted the PD-mimic phenotype, this time with no detectable side effects. BMPPB-32 also reduced the loss of vision in old flies [6]. Our next steps are to optimize the drug regimen, to test a range of doses of novel compounds, to determine their tissue specificity (e.g., nerve vs muscle vs fat) and to see if taking the drugs in young animals will provide long-lasting protection, or if the drugs need to be continuously supplied. Our current data show that we have clear PD-mimic in the fly, which can be treated with potential LRRK2 kinase inhibitors, in a fast, whole-organism functional assay, making a good experimental stepping stone.

\section{But is it on the road to the clinic?}

Or just an experimentally convenient diversion? Will drugs that work in flies also work in mammals and humans? Although flies are not 'little humans', they share many genes: about $60-70 \%$ of genes associated with human disease have homologues in flies [5]. The basic cellular metabolism is very similar in both organisms, and the function of the nervous system is highly conserved. This is well illustrated by our SSVEP experiments, which show a clear functional link in the processing of visual information between flies and humans. The parallel is strengthened by the fact that, in PD patients as well as flies, the loss of function in retinal dopamine neurons affects vision [9]. We have extra confidence that the fly provides a good PD-mimic, because there are reports in the older PD literature of tests with SSVEP-like approaches, finding visual defects from patients which closely resemble our data (e.g., [10]). However, other reports did not note a visual phenotype. We have speculated that this disparity may result from genetic differences between the people tested, so this needs to be revisited using a consistent SSVEP technique with fully genotyped patients. We conclude that our experiments therefore provide, not just a diversionary stepping stone, but a motorway bridge between experiments in vitro and novel therapies for the human patient. 


\section{Financial \& competing interests disclosure}

The authors are grateful to Lundbeck A/S, who supported their work with the supply of reagents gratis. The authors thank The Wellcome Trust (ref.: 097829/Z/11/A) and the York Open Access Fund for support. The authors have no other relevant affiliations or financial involvement with any organization or entity with a financial interest in or financial conflict with the subject matter or materials discussed in the manuscript apart from those disclosed.

\section{References}

1 Poewe W, Mahlknecht P. Pharmacological treatment of Parkinson's disease. In: Movement Disorders. Watts RL, Standaert DG, Obeso JA (Eds). McGraw-Hill, NY, USA, 271-304 (2011).

2 Singleton AB, Farrer MJ, Bonifati V. The genetics of Parkinson's disease: progress and therapeutic implications. Mov. Disord. 28(1), 14-23 (2013).

3 Greggio E, Cookson MR. Leucine-rich repeat kinase 2 mutations and Parkinson's disease: three questions. ASN Neuro. 1, e00002 (2009).

\section{No writing assistance was utilized in the production of}

this manuscript.

\section{Open access}

This article is distributed under the terms of the Creative Commons Attribution License 3.0 which permits any use, distribution, and reproduction in any medium, provided the original author(s) and the source are credited. To view a copy of the license, visit http://creativecommons.org/ licenses/by/3.0/

4 Beal M. Parkinson's disease: a model dilemma. Nature 466(August), S8-S10 (2010).

5 Cauchi RJ, van den Heuvel M. The fly as a model for neurodegenerative diseases: is it worth the jump? Neurodegener. Dis. 3(6), 338-356 (2006).

6 Afsari F, Christensen KV, Smith GP et al. Abnormal visual gain control in a Parkinson's disease model. Hum. Mol. Genet. 23 , 4465-4478 (2014).

7 Cajal SR, Sanchez D. [Contribution to the knowledge of the nervous system of insects. Part 1: Retina and optic lobes]. Trab. Lab. Invest. Bio. Univ. Madrid 13, 1-168 (1915) [translated].
8 Dopaminergic expression of the Parkinsonian gene LRRK2-G2019S leads to nonautonomous visual neurodegeneration, accelerated by increased neural demands for energy. Hum. Mol. Genet. 22, 2129-2140 (2013).

9 Archibald NK, Clarke MP, Mosimann UP, Burn DJ. The retina in Parkinson's disease. Brain 132(Pt 5), 1128-1145 (2009).

10 Bodis-Wollner I, Marx MS, Mitra S, Bobak P, Mylin L, Yahr M. Visual dysfunction in Parkinson's disease. Loss in spatiotemporal contrast sensitivity. Brain 110(6), 1675-1698 (1987). 